\title{
Development of compatible lignocellulolytic fungal consortium for rapid composting of rice straw.
}

\begin{abstract}
An experiment was conducted to evaluate the potential of lignocellulolytic fungi for rapid composting of rice straw. Forty-nine isolates of fungi were isolated from several natural and induced rice straw composting sources. Ten isolates were tested for their potential to decompose lignocellulosic rice straw by assessing their growth rate and biomass production, as well as their ability to decompose lignin and cellulose on rice-straw-powder-amended media. Four isolates (F26, F28, F29, and F44) were selected as potential lignocellulolytic agents for in-vitro compatibility study based on their optimum growth rate, biomass production, and lignocellulolytic activities. Six different interactions were found among four interacting isolates in the form of mutual intermingling, partial mutual intermingling, and inhibition at the contact point. Finally, a consortium of Aspergillus niger (F44) and Trichoderma viride (F26) was tested for in-vitro biodegradation of rice straw. The fungal consortium was able to decompose cellulose, hemicelluloses, lignin, and total carbon significantly $(\mathrm{p} \leq 0.05)$ over the control. The $\mathrm{C} / \mathrm{N}$ ratio was reduced to 19.5 from an initial value of 29.3 in three weeks of the biodegradation process, thus showing the potential of this method for use in large-scale composting of rice straw.
\end{abstract}

Keyword: Rice straw; Biodegradation; Lignocellulolytic; Fungal species. 